Vol 12, Issue 10, 2019

\title{
FORMULATION AND EVALUATION OF PH-DEPENDENT COLON-TARGETED TABLETS OF RIFAXIMIN BY DESIGN OF EXPERIMENT
}

\author{
RAWOOF MD ${ }^{1,2 *}$, RAJNARAYANA K ${ }^{2}$, AJITHA M ${ }^{3}$ \\ ${ }^{1}$ Department of Pharmaceutics, Jawaharlal Nehru Technological University, Kukatpally, Hyderabad, Telangana, India. ${ }^{2}$ Department of \\ Pharmaceutics, MAK College of Pharmacy, Ranga Reddy, Telangana, India. ${ }^{3}$ Centre for Pharmaceutical Sciences, Institute of Science and \\ Technology, Jawaharlal Nehru Technological University Hyderabad, Hyderabad, Telangana, India. Email: rawoofsucp@gmail.com
}

Received: 01 March 2019, Revised and Accepted: 06 September 2019

ABSTRACT

Objective: The research is designed at formulating and evaluating pH-sensitive rifaximin colon-targeted tablets for targeted action in proximal colon.

Method: The colon-targeted tablets are done by granulation of three levels of polymers such as Eudragit L30D, Carbopol 974P, and ethyl cellulose. The evaluation parameters such as swelling studies, drug dissolution, in vitro drug release studies, stability, and the Fourier transform infrared studies carried out for optimized formulations.

Results: Physicochemical parameters of all the 27 formulations (RF1-RF27) evaluated and RF21 is chosen for further investigation based on weight variation, hardness, drug content, and swelling index. The in vitro drug release studies indicate that the optimized formulation RF 21 released $98.75 \%$ drug within $24 \mathrm{~h}$. The stability studies indicate that the formulation is stable.

Conclusion: An effective and stable pH-dependent rifaximin colon-targeted tablet formulated for the targeted treatment of bowel syndrome.

Keywords: Rifaximin, Colon drug delivery system, Swelling index, Irritable bowel syndrome.

(C) 2019The Authors. Published by Innovare Academic Sciences Pvt Ltd. This is an open accessarticle under the CC BY license (http://creativecommons. org/licenses/by/4. 0/) DOI: http://dx.doi.org/10.22159/ajpcr.2019.v12i10.32804

\section{INTRODUCTION}

The drug delivery systems are aimed at supplying the drug to an object site in a body. Drug delivery systems alter drug dissolution profile for increasing the product efficacy [1]. Colon drug delivery system is targeted to deliver the drug to of gastrointestinal (GI) tract [2]. Conventional oral administrated drugs lack the special property of drug targeting to GI sites. The colon-targeted drug delivery systems are applied for the treatment of bowel diseases and colonic cancer. The colon-specific drug delivery systems (CDDSs) protect the drug till it reaches the colon, ensuring that the drug is not released in stomach or small intestine. They possess long retention time of 4-5 days, thus facilitating maximum adsorption of drug, hence, making it ideal for targeted delivery [3]. CDDS applied to reduce the dosing frequency of drug, to deliver the drug to regions that have less hostile metabolic, to increase the pharmacological activity, and to prevent drug from degradation [4].

CDDS depends on $\mathrm{pH}$ and spreadability of the drug, the delivery system, GI transits time and the interaction level between the same [5]. The release of drug in GI tract also depends on ability of enzymes to break bonds between drug and carrier molecules. CDDSs with maximum drug release are considered ideal for colon release.

Among various approaches, the less suitable method is $\mathrm{pH}$-dependent system due to variations in $\mathrm{pH}$ of GI tract. In spite of these intra- and inter-subject variations, these were considered better when combined with time-dependent systems [6].

Rifaximin is used in the treatment of hepatic encephalopathy, diarrhea, and bowel syndromes. Rifaximin acts by inhibiting RNA synthesis in susceptible bacteria by binding to the beta-subunit of bacterial deoxyribonucleic acid-dependent ribonucleic acid polymerase enzyme [7]. This blocks translocation steps that form phosphodiester bond. Rifaximin is selected as colon tablet due to its poor oral availability.

\section{EXPERIMENTATION}

Materials

Rifaximin was procured from Hetero Drugs Ltd., Hyderabad, India. Eudragit RL 100, HPMC K4M, and ethyl cellulose were purchased from Aurobindo Labs, India. All other chemicals and solvents were used without further purification.

\section{Instruments}

The formulations carried out using tablet compression machine (Cadmach, Ahmedabad) and dissolution apparatus (Electrolab [TDT- 08L], Mumbai). Chemical analysis was performed using Fourier transform infrared (FTIR) spectrophotometer (Shimadzu 8400S, Japan) and ultraviolet (UV)-visible spectrometer (Shimadzu, Japan).

Preparation of colon-targeted tablets of rifaximin Twenty-seven formulations (RF1-RF27) were prepared using polymers such as Eudragit L30D, Carbopol 974P, and ethyl cellulose. The $3^{3}$ response surface method adopted for design of experiment. The formulations with varied polymer concentration prepared. All the prepared formulations were constituted with magnesium stearate. The mixture was passed through sieve no 85. The polyvinylpyrrolidone $\mathrm{K} 30$ and $5 \%$ in isopropyl alcohol were chosen as binder solvent for granulation. The wet crude passed through sieve no $12 \#$. Contents dried for $2 \mathrm{~h}$ at $45^{\circ} \mathrm{C}$. The obtained material was compressed with $10 \mathrm{~mm}$ flat punch after screening through sieve no.18\#.

Evaluation of rifaximin colon-targeted tablets Weight variation test

The average weight of 20 tablets determined randomly. Each tablet weighed individual and checked for deviation from average weight. The percentage deviation tabulated [8]. 


\section{Thicknesses}

The thickness measured in millimeters using a screw gauge for 10 tablets. The average thickness calculated. The deviation of each of 10 tablets from average thickness noted as the standard deviation.

Hardness

The hardness is measured as tensile strength in $\mathrm{kg} / \mathrm{cm}^{2}$ by tablet hardness testing machine. Randomly selected three tablets and tested for hardness. The average of all three values calculated [9].

\section{Friability}

The Roche friabilator (Electrolab, India) is used for determining friability of tablets. Twenty tablets placed in machine and run for 100 revolutions. The tablets weighed after dusting [10].

The friability (F \%) is calculated by the formula,

$$
\mathrm{F} \%=\left(1-\mathrm{W}_{0} / \mathrm{W}\right) \times 100 \text {. }
$$

\section{Drug content}

The average weight of 20 tablets calculated. $10 \mathrm{mg}$ of tablet powder mixed with $100 \mathrm{ml}$ of phosphate buffer ( $\mathrm{pH} 7.2$ ) for complete dissolution. Contents filtered and filtrate checked for drug concentration at $227 \mathrm{~nm}$ by UV spectrometer.
In vitro swelling index

The pre-weighed tablet placed in $5 \mathrm{ml}$ of phosphate buffer ( $\mathrm{pH}$ 7.2) for $12 \mathrm{~h}$ tablet weight at intervals of $1,2,4,8,10$, and $12 \mathrm{~h}$ by taking it out from solvent using a filter paper [10]. The swelling index obtained using the following formula.

$$
\text { Swelling index }(\mathrm{S} . \mathrm{I})=(\mathrm{Wt}-\mathrm{Wo}) / \mathrm{Wo} \times 100 \text {, }
$$

Where, S.I=Swelling index,

$\mathrm{Wt}=$ Weight of tablet time $\mathrm{t}$

Wo=Initial weight.

\section{In vitro drug release study}

The dissolution of prepared tablet formulations was carried out by obeying various conditions. The dissolution medium chosen is combination of acidic buffer pH $1.2(2 \mathrm{H}$ ), phosphate buffer $\mathrm{pH} 6.8$ (4 $\mathrm{H})$, and phosphate buffer ( $\mathrm{pH} 7.2$ ) in $900 \mathrm{ml}$ dissolution medium. The drug content estimated at intervals of $1,2,3,4,6,8,10,12$, and $24 \mathrm{~h}$ by UV spectroscopy at $227 \mathrm{~nm}$ [11].

\section{Drug release kinetics}

The drug release data fitted into various linear and non-linear models and checked for linearity [12].

\begin{tabular}{|c|c|c|c|c|c|c|}
\hline F. No. & *Weight variation (mg) & \#Thickness (mm) & \#Hardness $\left(\mathrm{kg} / \mathrm{cm}^{2}\right)$ & \#Friability (\%) & \#Drug content (\%) & Swelling index (\%) \\
\hline RF1 & $801.65 \pm 1.2$ & $6.0 \pm 0.12$ & $6.5 \pm 0.12$ & $0.53 \pm 0.01$ & $96.23 \pm 0.63$ & $75 \pm 0.76$ \\
\hline RF2 & $798.69 \pm 0.8$ & $6.2 \pm 0.06$ & $6.4 \pm 0.06$ & $0.56 \pm 0.02$ & $96.04 \pm 0.06$ & $78 \pm 0.72$ \\
\hline RF3 & $798.04 \pm 0.5$ & $6.0 \pm 0.06$ & $6.3 \pm 0.06$ & $0.61 \pm 0.03$ & $97.56 \pm 0.14$ & $79 \pm 0.64$ \\
\hline RF4 & $801.05 \pm 0.0$ & $6.2 \pm 0.12$ & $6.1 \pm 0.12$ & $0.71 \pm 0.01$ & $97.11 \pm 1.01$ & $87 \pm 0.81$ \\
\hline RF5 & $801.54 \pm 0.4$ & $6.3 \pm 0.00$ & $6.2 \pm 0.00$ & $0.64 \pm 0.02$ & $95.23 \pm 0.8$ & $74 \pm 1.03$ \\
\hline RF6 & $800.78 \pm 0.4$ & $6.3 \pm 0.10$ & $6.8 \pm 0.06$ & $0.65 \pm 0.01$ & $96.45 \pm 0.31$ & $75 \pm 0.84$ \\
\hline RF7 & $800.65 \pm 0.3$ & $6.2 \pm 0.10$ & $6.4 \pm 0.10$ & $0.57 \pm 0.02$ & $95.18 \pm 0.49$ & $72 \pm 0.72$ \\
\hline RF9 & $800.76 \pm 0.35$ & $6.1 \pm 0.06$ & $6.5 \pm 0.06$ & $0.59 \pm 0.00$ & $97.13 \pm 0.56$ & $83 \pm 0.80$ \\
\hline RF10 & $800.49 \pm 0.2$ & $6.3 \pm 0.20$ & $6.1 \pm 0.42$ & $0.78 \pm 0.02$ & $96.23 \pm 0.24$ & $78 \pm 0.46$ \\
\hline RF11 & $801.53 \pm 0.4$ & $6.1 \pm 0.06$ & $6.3 \pm 0.06$ & $0.75 \pm 0.01$ & $98.97 \pm 0.21$ & $87 \pm 0.67$ \\
\hline RF12 & $802.58 \pm 0.3$ & $6.1 \pm 0.00$ & $6.5 \pm 0.06$ & $0.74 \pm 0.02$ & $96.45 \pm 0.76$ & $89 \pm 0.93$ \\
\hline RF13 & $801.34 \pm 0.2$ & $6.2 \pm 0.26$ & $6.7 \pm 0.35$ & $0.73 \pm 0.02$ & $98.45 \pm 0.48$ & $97 \pm 0.53$ \\
\hline RF14 & $798.67 \pm 0.3$ & $6.2 \pm 0.21$ & $6.5 \pm 0.21$ & $0.75 \pm 0.03$ & $97.98 \pm 0.23$ & $84 \pm 1.08$ \\
\hline RF15 & $799.65 \pm 0.2$ & $6.3 \pm 0.06$ & $6.8 \pm 0.23$ & $0.76 \pm 0.02$ & $96.45 \pm 0.36$ & $91 \pm 0.91$ \\
\hline RF16 & $800.65 \pm 0.3$ & $6.1 \pm 0.25$ & $6.5 \pm 0.23$ & $0.76 \pm 0.01$ & $95.45 \pm 0.69$ & $87 \pm 0.63$ \\
\hline RF18 & $801.87 \pm 0.1$ & $6.4 \pm 0.25$ & $6.6 \pm 0.35$ & $0.81 \pm 0.01$ & $96.56 \pm 0.23$ & $96 \pm 0.90$ \\
\hline RF19 & $799.67 \pm 0.3$ & $6.2 \pm 0.12$ & $6.3 \pm 0.12$ & $0.80 \pm 0.03$ & $97.29 \pm 0.34$ & $91 \pm 0.75$ \\
\hline RF20 & $799.32 \pm 0.2$ & $6.3 \pm 0.12$ & $6.4 \pm 0.2$ & $0.65 \pm 0.03$ & $98.18 \pm 0.81$ & $89 \pm 0.67$ \\
\hline RF21 & $800.27 \pm 0.4$ & $6.2 \pm 0.06$ & $6.3 \pm 0.06$ & $0.61 \pm 0.02$ & $99.48 \pm 0.69$ & $98 \pm 0.54$ \\
\hline RF22 & $800.27 \pm 0.1$ & $6.3 \pm 0.12$ & $6.2 \pm 0.12$ & $0.52 \pm 0.03$ & $97.18 \pm 0.07$ & $97 \pm 0.67$ \\
\hline RF23 & $800.26 \pm 0.13$ & $6.1 \pm 0.17$ & $6.7 \pm 0.4$ & $0.77 \pm 0.05$ & $98.14 \pm 0.76$ & $86 \pm 0.54$ \\
\hline RF24 & $800.10 \pm 0.5$ & $6.2 \pm 0.00$ & $6.8 \pm 0.23$ & $0.72 \pm 0.08$ & $98.16 \pm 0.12$ & $94 \pm 0.86$ \\
\hline RF25 & $799.12 \pm 0.6$ & $6.2 \pm 0.17$ & $6.7 \pm 0.12$ & $0.66 \pm 0.02$ & $97.23 \pm 0.00$ & $92 \pm 0.70$ \\
\hline RF26 & $800.16 \pm 0.8$ & $6.3 \pm 0.10$ & $6.5 \pm 0.21$ & $0.71 \pm 0.89$ & $97.78 \pm 0.23$ & $93 \pm 0.68$ \\
\hline RF27 & $800.29 \pm 0.15$ & $6.4 \pm 0.29$ & $6.4 \pm 0.45$ & $0.86 \pm 0.03$ & $98.10 \pm 0.40$ & $95 \pm 0.75$ \\
\hline
\end{tabular}

Table 1: Evaluation tests of rifaximin colon-targeted tablets

*Values are expressed in mean \pm standard deviation: $(n=20)$, \#Values are expressed in mean \pm standard deviation: $(n=3)$

\begin{tabular}{|c|c|c|c|c|c|c|c|c|}
\hline \multirow[t]{2}{*}{ Formulation code } & \multicolumn{2}{|c|}{ Zero order } & \multicolumn{2}{|c|}{ First order } & \multicolumn{2}{|c|}{ Higuchi } & \multicolumn{2}{|c|}{ Korsmeyer-Peppas } \\
\hline & $\mathbf{R}^{2}$ & $\mathbf{K}$ & $\mathbf{R}^{2}$ & $\mathbf{K}$ & $\mathbf{R}^{2}$ & K & $\mathbf{R}^{2}$ & $\mathbf{N}$ \\
\hline RF21 & 0.996 & 4.165 & 0.939 & 0.087 & 0.937 & 23.24 & 0.929 & 0.865 \\
\hline
\end{tabular}

Table 2: Release kinetics of optimized formulation of rifaximin colon-targeted tablets (RF21)

\begin{tabular}{|c|c|c|c|c|c|c|c|c|}
\hline \multirow[t]{2}{*}{ Formulation code } & \multicolumn{2}{|c|}{ Zero order } & \multicolumn{2}{|c|}{ First order } & \multicolumn{2}{|c|}{ Higuchi } & \multicolumn{2}{|c|}{ Korsmeyer-Peppas } \\
\hline & $\mathbf{R}^{2}$ & $\mathbf{K}$ & $\mathbf{R}^{2}$ & $\mathbf{K}$ & $\mathbf{R}^{2}$ & $\mathbf{K}$ & $\mathbf{R}^{2}$ & $\mathbf{N}$ \\
\hline Marketed formulation & 0.936 & 4.196 & 0.891 & 0.083 & 0.926 & 19.15 & 0.909 & 0.747 \\
\hline
\end{tabular}

Table 3: Release kinetics of marketed product 
Stability study

The stability of drug tested at $40^{\circ} \mathrm{C} \pm 2^{\circ} \mathrm{C} / 75 \% \mathrm{RH} \pm 5 \% \mathrm{RH}$ for 3 months using stability chamber. Sample stability checked at $0,30,60$, and 90 days period according to ICH guidelines [13].

\section{RESULTS AND DISCUSSION}

The preliminary drug authentication was carried out by UV spectrophotometry. The pure rifaximin exhibited absorption maxima at $227 \mathrm{~nm}$. Standard calibration curves of the drug plotted in acidic buffers of $\mathrm{pH} 1.2,6.8$, and 7.2 to check the stability of drug in respective condition. The results show that the graphs are linear over $2-12 \mu \mathrm{g} / \mathrm{ml}$ with $\mathrm{R}^{2}=0.999$ indicating stability of the drug.

Initially, 27 formulations of rifaximin (RF1-RF27) were prepared by wet granulation using three variables and three levels of polymers of different Eudragit L30D, Carbopol 974P, and ethyl cellulose (low, middle, and high concentrations) by design of experiment.

\section{Evaluation parameters of rifaximin colon-targeted tablets}

The rifaximin colon-targeted tablets were prepared by varying proportions of polymers by wet granulation method and further

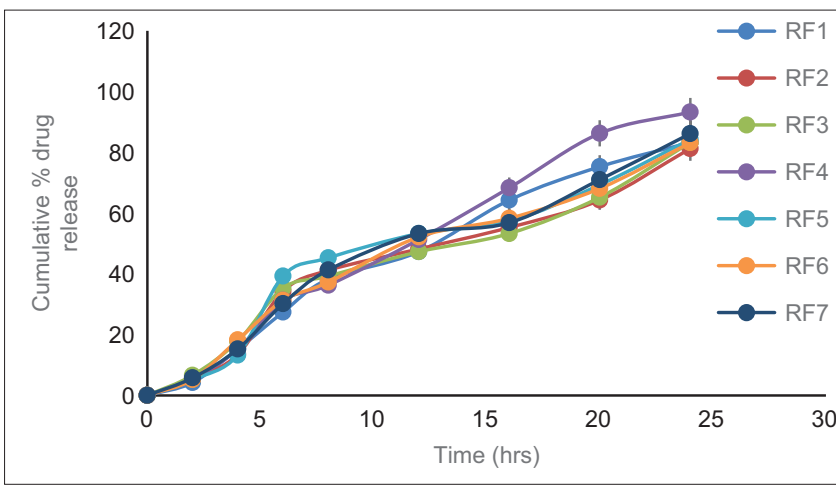

Fig. 1: In vitro drug release profile for rifaximin colon-targeted tablets RF1-RF7

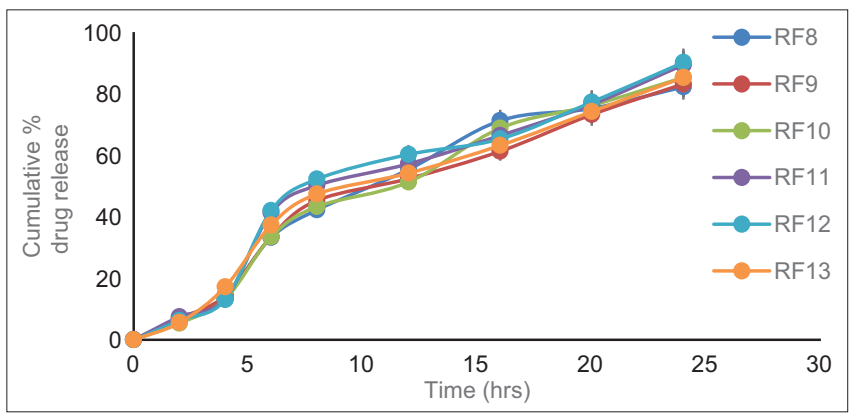

Fig. 2: In vitro drug release profile for rifaximin colon-targeted tablets RF8-RF13

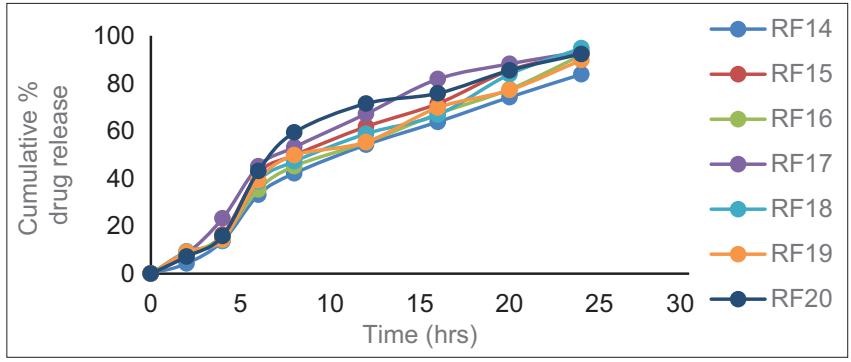

Fig. 3: In vitro drug release profile for rifaximin colon-targeted tablets RF14-RF20 evaluated for following evaluation tests. The results are found to be within the pharmacopoeia limits (Table 1).

The weight variations of all formulations are in the limit allowed that is $\pm 5 \%$ of total tablet weight. The crushing strength of fabricated tablets is between 6.0 and $7.0 \mathrm{~kg} / \mathrm{cm}^{2}$. The thickness of all the formulations is between the ranges of 6 and $6.4 \mathrm{~mm}$. The friability was found to be between 0.52 and $0.8 \%$. The drug content of all formulations is in between 95.18 and $99.48 \%$, indicating even distribution of drug in all the formulations. The swelling index of the tablet increases with increase in time up to $12 \mathrm{~h}$ indicating the drug remains in intestinal region until the drug is released completely from the delivery system and promotes evacuation after its release.

\section{In vitro drug release studies}

The release of rifaximin from different formulations is recorded in Figs. 1-4. The studies extended up to $24 \mathrm{~h}$. The highest drug release of 98.75\% found in RF21 of within $24 \mathrm{~h}$. Hence, RF21 is chosen for further investigations. The drug release of the marketed product is $82.23 \%$ up to $24 \mathrm{~h}$. A comparative in vitro study plot of optimized formulation (RF21) and marketed tablet (conventional and sustained) was recorded (Fig. 5).

In vitro drug release kinetics for optimized (RF21) formulation and marketed product

Drug release kinetics was evaluated for optimized formula of rifaximin colon-targeted tablets (RF21) and marketed formula (Table 2). The regression coefficient of 0.996 for zero-order plot, i.e., indicates zero-order mechanism for the drug release. Further, $n$ value obtained from the Korsmeyer-Peppas plots, i.e., 0.865 indicating non-Fickian (anomalous) transport. The marketed conventional formulation also followed the zero-order kinetics (Table 3).

\section{Design of experiment}

This method is mainly used to explain the effect of each factor on other factors, whether this effect is significant or not, if significant how it influences the response. In this present work, the effect of ethyl cellulose on other two Eudragit L30D and Carbopol 974P is explained.

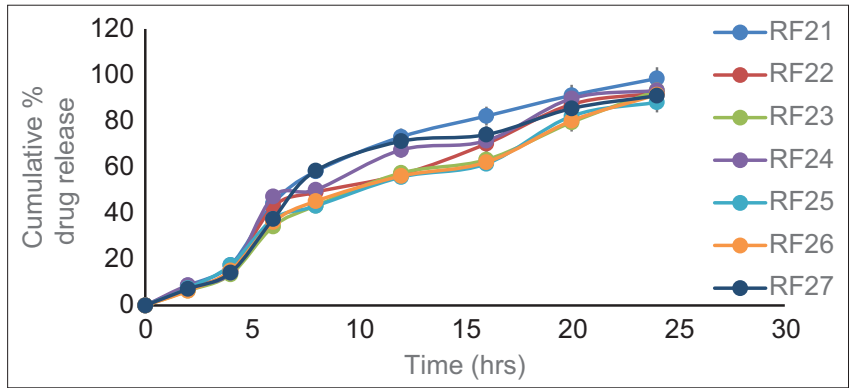

Fig. 4: In vitro drug release profile for rifaximin colon-targeted tablets RF21-RF27

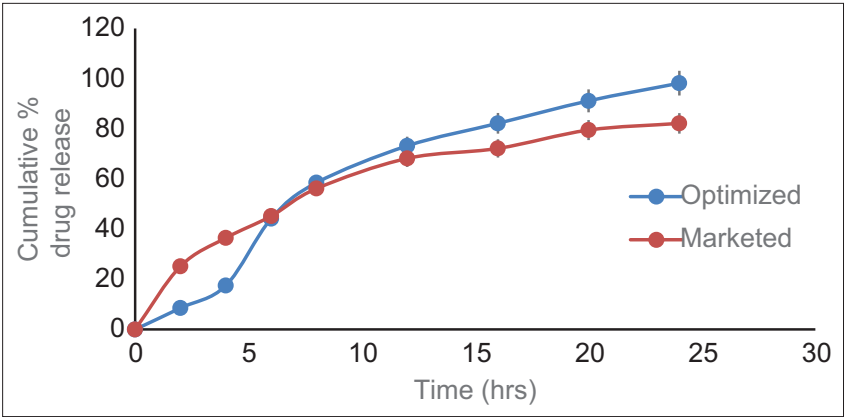

Fig. 5: Comparative In vitro study plot of rifaximin colon-targeted tablets optimized formulation (RF21) and conventional marketed tablet 
Table 4: Parameters after accelerated stability study of rifaximin colon-targeted tablet formulation (RF21)

\begin{tabular}{|c|c|c|c|c|}
\hline \multirow[t]{2}{*}{ Parameters } & \multicolumn{4}{|c|}{ Temperature maintained at $40 \pm 2^{\circ} \mathrm{C}$; $\mathrm{RH}$ maintained at $75 \pm 5 \% \mathrm{RH}$} \\
\hline & Initial & After 1 month & After 2 months & After 3 months \\
\hline Drug content (\%) & $99.48 \pm 0.69$ & $98.46 \pm 0.65$ & $98.33 \pm 0.37$ & $98.32 \pm 0.22$ \\
\hline Swelling index (\%) & $98 \pm 0.54$ & $98.0 \pm 0.46$ & $98.0 \pm 0.37$ & $98.0 \pm 0.23$ \\
\hline Hardness $\left(\mathrm{Kg} / \mathrm{cm}^{2}\right)$ & $6.3 \pm 0.66$ & $6.3 \pm 0.58$ & $6.3 \pm 0.45$ & $6.3 \pm 0.37$ \\
\hline
\end{tabular}

RH: Relative humidity

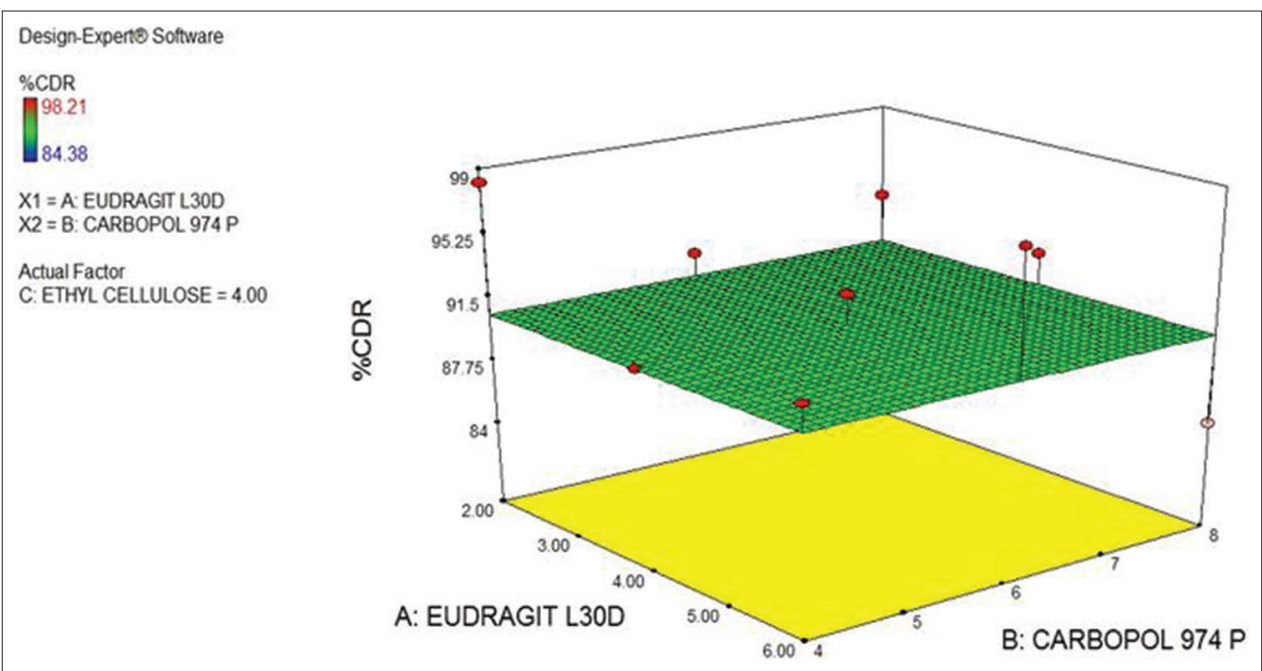

Fig. 6: Response surface plot showing the influence of amount of polymer on the release profile of rifaximin colon tablets for cumulative percentage drug released

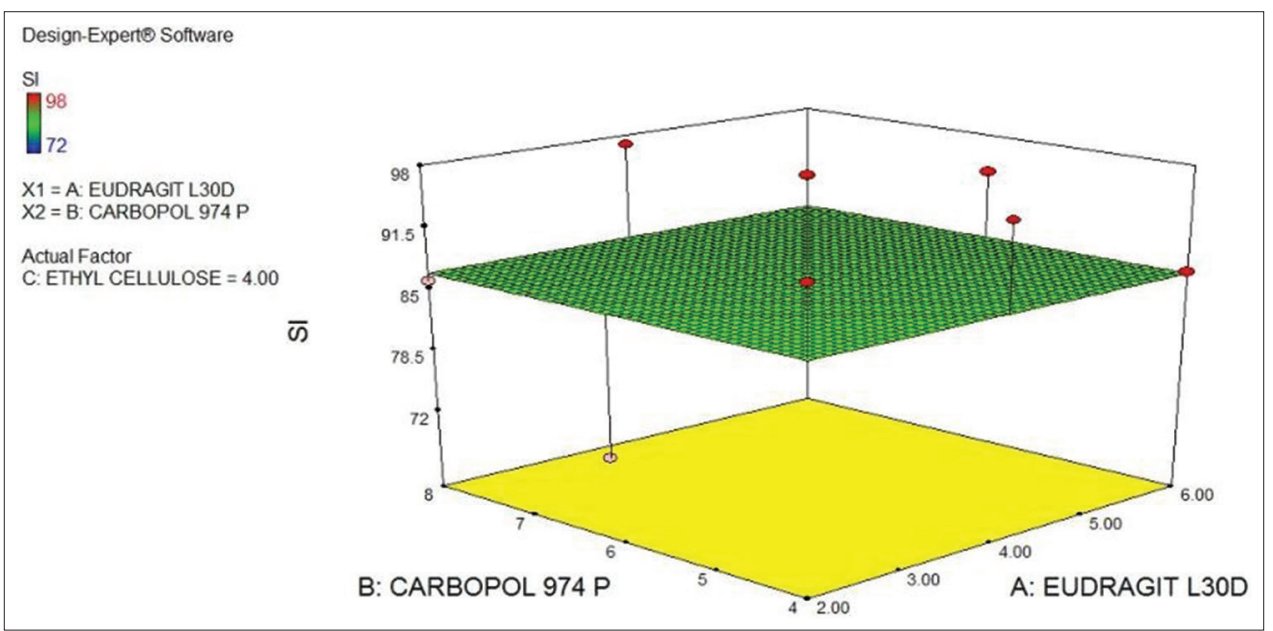

Fig. 7: Response surface plot showing the influence of amount of polymer on swelling index of rifaximin

In Fig. 6, the effect of ethyl cellulose on percentage cumulative drug release shows significant effect of ethyl cellulose on percentage cumulative drug release. The percentage cumulative drug release of all the three formulations is in between 84.38 and $98.21 \%$ but when ethyl cellulose is in low concentration, the maximum percentage cooldown reduction is near $84.38 \%$. This is the effect of factor (ethyl cellulose) on response which is a negligible on swelling index as formulations exhibit excellent swelling property and there is slightly influence on swelling index by ethyl cellulose (Fig. 7). Overlay plot of rifaximin colon-targeted tablets is represented in Fig. 8.

\section{Drug-excipient compatibility studies}

FTIR studies spectroscopy

The FTIR spectrum of rifaximin and optimized formulation is shown in Figs. 9 and 10. The $\mathrm{O}-\mathrm{H}$ and $\mathrm{C}=\mathrm{O}$ stretching in rifaximin pure drug were observed at 3448 and $1745 \mathrm{~cm}^{-1}$, respectively, which remained intact in physical mixture of rifaximin and excipients. C-H stretching of pure drug is at $2924 \mathrm{~cm}^{-1}$, whereas it shows the same peak for physical mixture also. C-H bending of pure drug and physical mixture is at 790 $\mathrm{cm}^{-1}$. The FTIR spectrum of rifaximin optimized formulation RF21 also exhibited characteristic bands 3437 and $1786 \mathrm{~cm}^{-1}$ for $\mathrm{O}-\mathrm{H}$ and $\mathrm{C}=\mathrm{O}$ stretching and $2924 \mathrm{~cm}^{-1}$ for C-H stretching, and $790 \mathrm{~cm}^{-1}$ for C-H bending) consistent with the molecular structure of rifaximin pure drug which indicated that no chemical interaction occurred between the drug and excipients used in the formulation.

\section{Stability studies}

The stability studies of the optimized formulation (RF21) indicated no significant effect on drug release, hardness, drug content, and swelling study data. Hence, the formulations are stable (Table 4). 


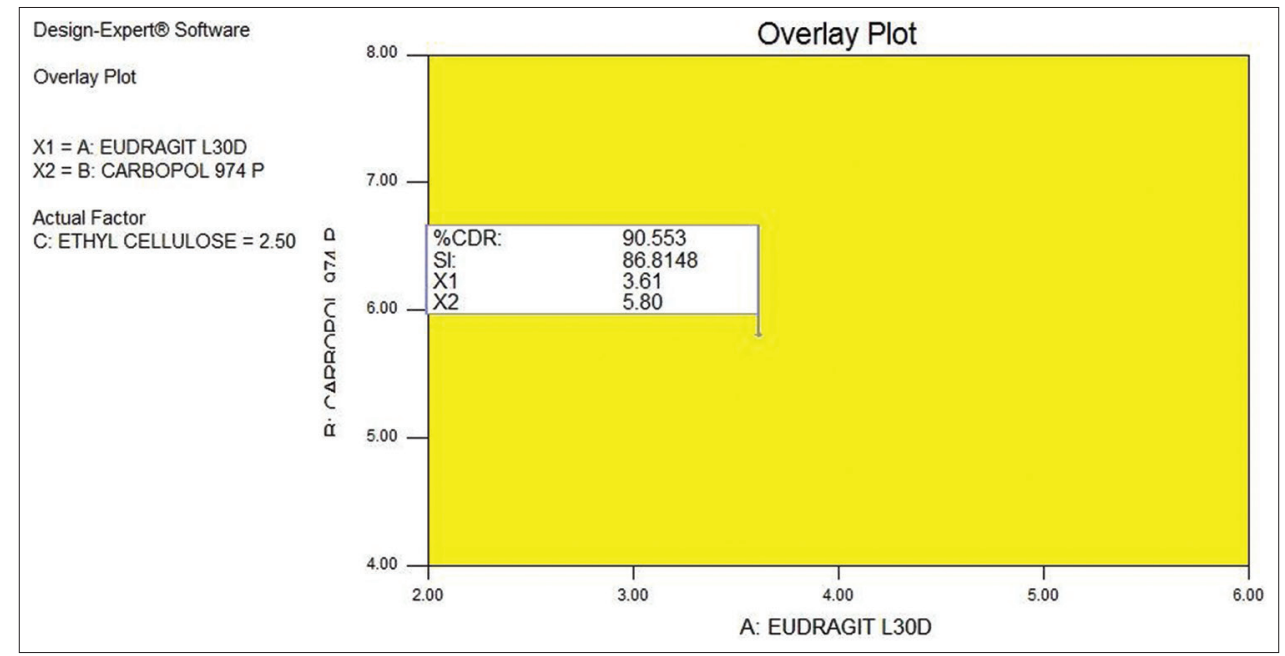

Fig. 8: Overlay plot of rifaximin colon-targeted tablets

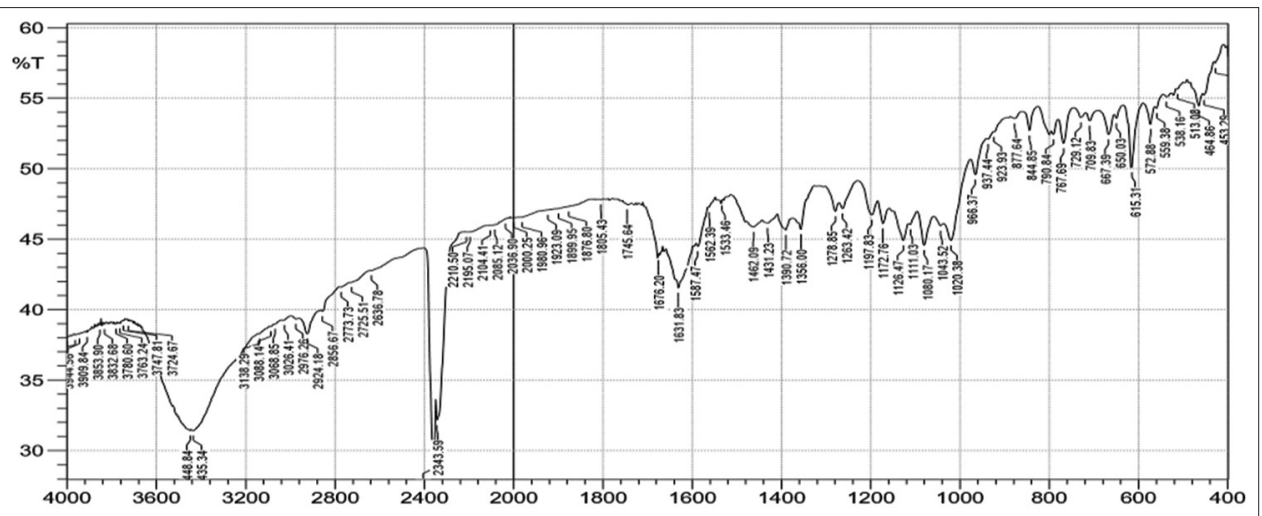

Fig. 9: Fourier transform infrared spectrum of pure drug rifaximin

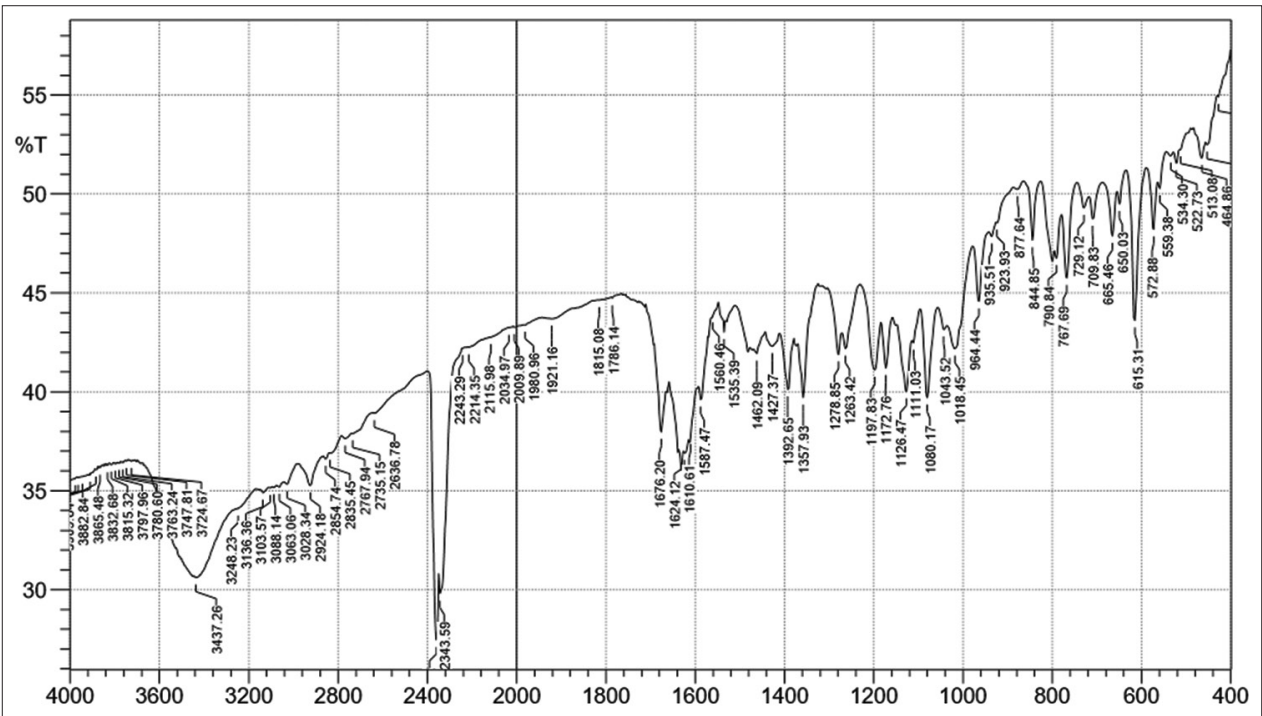

Fig. 10: Fourier transform infrared spectrum of optimized formulation of rifaximin colon-targeted tablets

\section{CONCLUSION}

The present study is conducted to formulate pH-sensitive matrix tablets of rifaximin for targeted action in proximal colon. Twenty-seven formulations of rifaximin (RF1-RF27) were prepared using three levels of polymers of different Eudragit L30D, Carbopol 974P, and ethyl cellulose (low, middle, and high concentrations) by design of experiments software. The results of physicochemical parameter test applied on all the formulations designated that the weight variations are within allowed limit. The drug content measured for formulations indicates uniform drug distribution. Swelling index indicates that drug will remain in intestinal region until the drug is released completely from the delivery system and promotes evacuation after its release. Drug compatibility studies carried out by recording the FTIR spectra indicate no chemical interaction between the drug and the excipients used. In vitro drug 
release of colon-targeted rifaximin tablets studied up to $24 \mathrm{~h}$. The highest drug release of $98.75 \%$ found in the RF21 at within 24 h. Drug release kinetics for optimized formulation RF21 indicates that it follows zeroorder mechanism. The stability studies indicate no major changes in swelling index, hardness, drug content, and in vitro drug release.

\section{CONFLICTS OF INTEREST}

No conflicts of interest by authors.

\section{AUTHORS' CONTRIBUTIONS}

All authors contributed equally.

\section{REFERENCES}

1. Philip AK, Philip B. Colon targeted drug delivery systems: A review on primary and novel approaches. Oman Med J 2010;25:79-87.

2. Philip AK, Dabas S, Pathak K. Optimized prodrug approach: A means for achieving enhanced anti-inflammatory potential in experimentally induced colitis. J Drug Target 2009; 17:235-41.

3. Odeku OA, Fell JT. In vitro evaluation of Khaya and Albizia gums as compression coatings for drug targeting to the colon. J Pharm Pharmacol 2005;57:163-8.

4. Akala EO, Elekwachi O, Chase V, Johnson H, Lazarre M, Scott K, et al. Organic redox-initiated polymerization process for the fabrication of hydrogels for colon-specific drug delivery. Drug Dev Ind Pharm 2003;29:375-86.

5. Chourasia MK, Jain SK. Pharmaceutical approaches to colon targeted drug delivery systems. J Pharm Pharm Sci 2003;6:33-66.

6. Singh SNC, Mahato AK. Formulation and evaluation of metronidazole tableted microspheres for colon drug delivery. Asian J Pharm Clin Res 2016;9:398-403

7. Upadhyay S, Agrawal P, Bansal M, Gupta A. Rifaximin induced hyponatremia: A case report. Asian J Pharm Clin Res 2016;9:1.

8. Gazzaniga A, Bussetti C, Moro L, Sangali ME, Giordano F. Time dependent oral delivery system for colon targeting. STP Pharm Sci 1995;5:83-8.

9. Raosaheb S, Shendge, Fatima J. Development of colon specific drug delivery of aceclofenac by using effective binder system of ethyl cellulose. Int J Biomed Pharm Sci 2010;3:1-5.

10. Mayur M, Santnu LP. A synchronous colon specific drug delivery system for orally administered mesalamine. Acta Pharm Sci 2009;51:251-60.

11. Salerno C, Carlucci AM, Bregni C. Study of in vitro drug release and percutaneous absorption of fluconazole from topical dosage forms. AAPS PharmSciTech 2010;11:986-93.

12. Baishya H. Application of mathematical models in drug release kinetics of carbidopa and levodopa ER tablets. J Dev Drugs 2017;6:1-4.

13. Brown $\mathrm{G}$. The value of drug stability studies and their publication. Can J Hosp Pharm 2018;71:161-2. 